\title{
The resolution theorems for intuitionistic fuzzy sets with sheet-level sets
}

\section{Arif Bal, Gökhan Çuvalcoğlu and Gül Tümen}

\author{
Department of Mathematics, Mersin University \\ 33016, Mersin Turkey \\ e-mails: arif.bal .math@gmail.com, gcuvalcioglu@gmail.com, \\ gultumenn@gmail.com
}

Received: 20 March 2019

Revised: 15 April 2019

Accepted: 24 April 2019

\begin{abstract}
In this study, the resolution theorems for IFSs were given by using the $\alpha-t$ sheet level set and strict $\alpha-t$ sheet level set of IFSs. For the second resolution theorem, the definition of strict $\alpha-t$ sheet level set of IFSs was given in this paper. Because of the nature of IFSs, one might give those theorems using fuzzy sets. In this paper, that problem was solved.
\end{abstract}

Keywords: Intuitionistic fuzzy sets, IFS Sheet $t$-level, IFS $\alpha-t$ sheet level and intuitionistic fuzzy $t$-subset, Resolution theorems.

2010 Mathematics Subject Classification: 03E72, 03E99.

\section{Introduction}

The concept of fuzzy sets which is extension of crisp set by expanding the membership degree of an element in an arbitrary universal set from $\{0,1\}$ to the real unit interval $[0,1]$, was introduced by Zadeh [7]. Let $X$ be a universal set, then a fuzzy set over $X$ is a function $\mu: X \rightarrow[0,1]$. A fuzzy set on $X$ shortly is denoted by $F S(X)$. For $x \in X$, the value of $\mu(x)$ is called the membership degree of $x$ and $1-\mu(x)$ is called the non-membership degree of $x$ where the plus of membership degree and non-membership degree of an element always is 1 .

The concept of intuitionistic fuzzy sets which is one of the extension of fuzzy sets, is introduced by Atanassov [1]. Intuitionistic fuzzy sets are defined from an universe to lattice $[0,1] \times[0,1]$. An intuitionistic fuzzy sets on $X$ shortly is denoted by $\operatorname{IFS}(X)$. Let $X$ be a universal set, then an intuitionistic fuzzy set over $X$ consists of functions $\mu: X \rightarrow[0,1]$ and $v: X \rightarrow[0,1]$ where; for $x \in X$, the value of $\mu(x)$ is called the membership degree of $x$ and $\nu(x)$ is called the 
non-membership degree of $x$, the plus of $\mu(x)$ and $v(x)$ is equal to 1 or less than 1 and the hesitation degree of $x$ is $1-(\mu(x)+\nu(x))$.

The concept of level sets on fuzzy sets was originally introduced by Zadeh [7]. It is possible to descend from fuzzy sets to the classical sets and get some properties for the fuzzy sets by using level sets. The level sets help us to extend the operations and structures defined on crisp sets to the case of fuzzy sets [3].

Atanassov extended the idea of level sets from fuzzy sets to intuitionistic fuzzy sets [2]. The level set of any intuitionistic fuzzy set is also a crisp set, as for fuzzy sets [6]. The different types of level set of intuitionistic fuzzy sets were given by G. Çuvalcıoğlu [4]. In contrast with the other definition, level sets were defined as $s$-fuzzy sets. In this way, one can say that a level set of any intuitionistic fuzzy set need not be a classical set, but the level of it is classical set.

\section{$2 \quad \alpha-t$ fuzzy sheet level sets of intuitionistic fuzzy sets}

In this section, some definitions and properties for intuitionistic fuzzy sets concerning level sets are given. The definition of level set for IFSs is the following,

Definition 1: [2] Let $A \in \operatorname{IFS}(X)$, then $(\alpha, \beta)$-level of $A$ and strong $(\alpha, \beta)$-level of $A$ are crisp subsets of the $X$, respectively, and are given by

$$
\begin{aligned}
& C_{(\alpha, \beta)}(A)=\left\{x \mid x \in X \text { such that } \mu_{A}(x) \geq \alpha, v_{A}(x) \leq \beta\right\}, \\
& C_{<\alpha, \beta>}(A)=\left\{x \mid x \in X \text { such that } \mu_{A}(x)>\alpha, v_{A}(x)<\beta\right\},
\end{aligned}
$$

where $\alpha, \beta \in[0,1]$ with $\alpha+\beta \leq 1$.

Definition 2: [4] Let $X$ be a set and $\mathrm{A}=\left\{\left\langle x, \mu_{A}(x), v_{A}(x)\right\rangle \mid x \in X\right\} \in \operatorname{IFS}(X)$. If $t \in[0,1]$ then sheet t-level of $A$ is defined as following

$$
A(t)=\left\{<x, \mu_{A}(x), v_{A}(x)>\mid \mu_{A}(x)+v_{A}(x)=t, x \in X\right\} .
$$

For $\forall t \in[0,1]$, the sheet $t$-level of an intuitionistic fuzzy set denotes discrete sheets. Each sheet $t$-level sets can be specified as a fuzzy set. From the above definition, it is easily seen that for every $t \in[0,1], A(t) \in F S(X)$. Because $A(t): X \rightarrow[0, t]$ and $[0, t] \sim[0,1]$. For short notation, $A(t)$ will be called $s$-fuzzy set on $X$, i.e. $\left.A(t) \in F S_{S}(X)\right)$.

Proposition 1: [4] Let $X$ be a set and A, B $\in$ IFS(X). For every $t \in[0,1]$ :

1. $(A \sqcup B)(t)=A(t) \sqcup B(t)$,

2. $A(t) \sqcap B(t)=(A \sqcap B)(t)$.

Proposition 2: [4] Let $X$ be a set and $A \in \operatorname{IFS}(X)$. If $t, s \in[0,1]$ then either $A(t) \sqcap A(s)=\varnothing$ or $t=s$.

Definition 3: [4] Let $X$ be a set and $A \in \operatorname{IFS}(X)$. If $t \in[0,1]$ and $\alpha \in[0, t]$ then

$$
A(t)_{\alpha}=\{x \mid x \in X, A(t)(x) \geq(\alpha, t-\alpha)\}
$$

is called $\alpha-t$ sheet level of $A$.

It can be easily seen from above definition that $A(t)_{\alpha}$ is a crisp set. First, we obtained a $t$-fuzzy set by $A(t)$ and then it is reduced to the $A(t)_{\alpha}$ crisp set. Some special cases are as follows. 
Proposition 3: [4] Let $X$ be a set and $A \in \operatorname{IFS}(X)$. If $t \in[0,1]$ then

$$
A(t)_{t}=\left\{x \mid x \in X, \mu_{A}(x)=t \wedge v_{A}(x)=0\right\}
$$

To illustrate the difference among the different definition of level sets for the intuitionistic fuzzy sets, we can examine the following example.

Example 1: [5] Let $X=\{a, b, c, d, e\}$ and

$$
A=\{(a, 0.5,0.4),(b, 0.2,0.3),(c, 0.5,0.3),(d, 0.4,0.4),(e, 0.4,0.1)\}
$$

$A(0.5)_{(0.3)}=\{e\}$ but $C_{(0.3,0.5)}(A)=\{a, c, d, e\}$ and $C_{(0.5,0.3)}(A)=\{c\}$.

$A(0.8)_{(0.5)}=\{c\}$ but $0.8+0.5>1$ so, we cannot obtain $C_{(0.8,0.5)}(A)$ or $C_{(0.5,0.8)}(A)$

From the example, $(\alpha, \beta)$-level of an intuitionistic fuzzy set $A$ and $\alpha-t$ sheet level of $A$ are different sets. For all $t \in[0,1]$ and $\alpha \in[0, t]$, we can determine $\alpha-t$ sheet level of $A$, if $\alpha+t>1$, then we cannot determine $(\alpha, \beta)$-level of $A$. Consequently, $\alpha-t$ sheet level of an intuitionistic fuzzy set allows a more extensive studying area.

Proposition 4: [4] Let $X$ be a set and $A \in \operatorname{IFS}(X)$. If $t \in[0,1]$ and $\alpha, \beta \in[0, t]$ such that $\alpha \leq \beta$ then

$$
A(t)_{\beta} \subseteq A(t)_{\alpha}
$$

Proposition 5: [4] Let $X$ be a set and $A, B \in I F S(X)$. If $t \in[0,1]$ and $\alpha \in[0, t]$ then:

1. $A(t)_{\alpha} \cup B(t)_{\alpha}=(A(t) \sqcup B(t))_{\alpha}$

2. $A(t)_{\alpha} \cap B(t)_{\alpha}=(A(t) \sqcap B(t))_{\alpha}$

3. $A(t)_{\alpha}{ }^{c}=t-A(t)_{\alpha},(t(x)=t)$

Theorem 1: [4] Let $X$ be a set and $A \in \operatorname{IFS}(X)$. For t, $s \in[0,1]$ and $\alpha \in[0, t], \beta \in[0, s]$ if $\alpha \leq s$ then:

1. $A(t)_{\alpha} \cup A(t)_{\beta}=A(t)_{\min (\alpha, \beta)}$

2. $A(t)_{\alpha} \cap A(t)_{\beta}=A(t)_{\max (\alpha, \beta)}$

Remark: [4] In fuzzy set theory union and intersection of two level set is again level set of the fuzzy set.

\section{The resolution theorems for IFSs}

The resolution theorems were studied by several author [7]. For intuitionistic fuzzy sets, it can be given using same idea with $\alpha-t$ sheet levels and fuzzy $t$-sets.

Theorem 2: (First Resolution Theorem) Let $X$ be universal and $A \in \operatorname{IFS}(X)$ then

$$
A=\bigcup_{\lambda_{1}+\lambda_{2}=\lambda} \bigcup_{t \in[0, \lambda]}\left(\lambda_{1}, \lambda_{2}\right)\left(t A(\lambda)_{t}\right)
$$

Proof: Let $x \in X$ and $A(x)=(\mathrm{a}, \lambda-\mathrm{a})$ then we get,

$\left(\mathrm{U}_{\lambda_{1}+\lambda_{2}=\lambda} \mathrm{U}_{t \in[0, \lambda]}\left(\lambda_{1}, \lambda_{2}\right)\left(t A(\lambda)_{t}\right)\right)(x)=\left(\mathrm{V}_{\lambda_{1}+\lambda_{2}=\lambda} \mathrm{V}_{t \in[0, \lambda]}\left(\lambda_{1}, \lambda_{2}\right)\left(t A(\lambda)_{t}\right)(x)\right.$

From the definition of $t A(\lambda)_{t}=t \wedge \chi_{A(\lambda)_{t}}$, we get $\left(t \wedge \chi_{A(\lambda)_{t}}\right)(x)=(t, \lambda-t) \wedge\left\{\begin{array}{l}(\lambda, 0), x \in A(\lambda)_{t} \\ (0, \lambda), x \notin A(\lambda)_{t}\end{array}\right.$ 
If we use this equality, we get

$$
\begin{aligned}
& \left(\mathrm{U}_{\lambda_{1}+\lambda_{2}=\lambda} \mathrm{U}_{t>a}\left(\lambda_{1}, \lambda_{2}\right)\left(t A(\lambda)_{t}\right)\right)(x) \\
& =\left(\mathrm{V}_{\lambda_{1}+\lambda_{2}=\lambda} \mathrm{V}_{t \in[0, \lambda]}\left(\lambda_{1}, \lambda_{2}\right)\left(t A(\lambda)_{t}\right)(x)\right. \\
& =\left(\mathrm{V}_{\lambda_{1}+\lambda_{2}=\lambda} \mathrm{V}_{t<a, t \in[0, \lambda]}\left(\lambda_{1}, \lambda_{2}\right) \wedge(t, \lambda-t) \wedge \chi_{A(\lambda)_{t}}(x)\right. \\
& \vee \mathrm{V}_{\lambda_{1}+\lambda_{2}=\lambda}\left(\lambda_{1}, \lambda_{2}\right) \wedge(a, \lambda-a) \wedge \chi_{A(\lambda)_{a}}(x) \\
& \vee\left(\mathrm{V}_{\lambda_{1}+\lambda_{2}=\lambda} \mathrm{V}_{k>a}\left(\lambda_{1}, \lambda_{2}\right) \wedge(k, \lambda-k) \wedge \chi_{A(\lambda)_{k}}(x)\right. \\
& =(0,1) \vee \mathrm{V}_{\lambda_{1}+\lambda_{2}=\lambda}\left(\lambda_{1}, \lambda_{2}\right) \wedge(a, \lambda-a) \wedge \chi_{A(\lambda)_{a}}(x) \vee(0,1) \\
& =(a, \lambda-a) \\
& =A(x) .
\end{aligned}
$$

After the following definition, we will give the second resolution theorem for IFS.

Definition 4: Let $X$ be a set and $A \in \operatorname{IFS}(X)$. If $t \in[0,1]$ and $\alpha \in[0, t]$ then

$$
A(t)_{<\alpha>}=\{x \mid x \in X, A(t)(x)>(\alpha, t-\alpha)\}
$$

is called strict $\alpha-t$ sheet level of $A$.

From above definition, it is seen easily that $A(t)_{<\alpha>}$ is a crisp set. By the above definition, the following theorem about resolution of IFSs can be given as the first resolution theorem.

Theorem 3: (Second Resolution Theorem) Let $X$ be universal and $A \in \operatorname{IFS}(X)$ then

$$
A=\bigcup_{\lambda_{1}+\lambda_{2}=\lambda} \bigcup_{t \in[0, \lambda]}\left(\lambda_{1}, \lambda_{2}\right)\left(t A(\lambda)_{<t>}\right)
$$

Proof: The proof is clear if we consider the classical property of supremum.

\section{Conclusion}

By the resolution theorems using sheet level sets, one can say that any intuitionistic fuzzy set can be characterized by all of its sheet $\alpha-t$ sheet level sets which are $t$-fuzzy sets. This is important for the nature and claim of IFSs. Because, it is known that IFSs are a generalization of fuzzy sets. Only for using for Second Resolution Theorem, we defined a new set "Strict $\alpha-t$ sheet level set for IFSs". But, it was not examined any property of them. Strict $\alpha-t$ sheet level set's properties are going to study.

\section{References}

[1] Atanassov K. T. (1983). Intuitionistic Fuzzy Sets, VII ITKR's Session, Sofia, June 1983 (Deposed in Centr. Sci.-Techn. Library of the Bulg. Acad. of Sci., 1697/84, in Bulgarian). Reprinted: Int. J. Bioautomation, 2016, 20 (S1), S1-S6.

[2] Atanassov K. T. (1999). Intuitionistic Fuzzy Sets: Theory and Applications, Heidelberg, Springer. 
[3] Çuvalcıŏlu, G., \& Yılmaz, S. (2014). Some properties of intuitionistic fuzzy equivalence relations and class trees w.r.t. intuitionistic fuzzy equivalence relations, Adv. Studies Contemp. Math., 24 (4), 77-86.

[4] Çuvalcıoglu, G. \& Tarsuslu (Yılmaz), S. The block and sheet levels of intuitionistic fuzzy sets, Journal of Universal Mathematics, (under review).

[5] Çuvalcıŏlu, G., Tarsuslu (Yılmaz), S., \& Demirbaş, E. (2019). Sheet level sets and representation theorem for intuitionistic fuzzy sets, Notes on Intuitionistic Fuzzy Sets, 25 (2), 15-19.

[6] Maheswari, A., Karthikeyan S. \& Palanivelrajan, M. (2012). Characterization of level sets of intuitionistic L-fuzzy semi filter of lattices, Notes on Intuitionistic Fuzzy Sets, 18 (2), $21-25$.

[7] Zadeh, L. A. (1965). Fuzzy sets, Information and Control, 8, 338-353. 